\title{
Neurologic outcome after aortic arch repair with frozen elephant trunk: The pivotal role of hypothermic circulatory arrest time
}

\author{
Mostafa Mousavizadeh ${ }^{1}$, Mohamad Bashir ${ }^{2}$, Mohammed Idhrees $^{3}$, Mahdi Daliri ${ }^{1}$, Hadi Abo \\ Aljadayel $^{1}$, Yousef Rezaei ${ }^{4}$, and Saeid Hosseini ${ }^{1}$ \\ ${ }^{1}$ Rajaie Cardiovascular Medical and Research Center \\ ${ }^{2}$ NHS Wales Health Education and Improvement Wales \\ ${ }^{3} \mathrm{SRM}$ Institutes for Medical Science Vadapalani \\ ${ }^{4}$ Affiliation not available
}

June 20, 2021

\begin{abstract}
This is response to a letter, so it does not contain structured abstract.
\end{abstract}

Neurologic outcome after aortic arch repair with frozen elephant trunk: The pivotal role of hypothermic circulatory arrest time

Mostafa Mousavizadeh ${ }^{1}$, MD; Mohamad Bashir ${ }^{*}$, M.D, Ph.D, MRCS

; Idhrees Mohammed ${ }^{3}$, MD; Mahdi Daliri ${ }^{1}$, MD; Hadi Abo Aljadayel ${ }^{1}$, MD;; Yousef Rezaei ${ }^{1}$, MD; Saeid Hosseini $^{1}$, MD

${ }^{1}$ Heart Valve Disease Research Center, Rajaie Cardiovascular Medical and Research Center, Iran University of Medical Sciences, Tehran, Iran

${ }^{2}$ Health Education \& Improvement Wales,Wales, UK

${ }^{3}$ Institute of Cardiac and Aortic Disorders (ICAD), SRM Institutes for Medical Science (SIMS Hospital), Chennai, Tamil Nadu, India

Running title:Frozen elephant trunk in acute type A dissection

* Corresponding Author:

Mohamad Bashir, MD, PhD, MRCS

Health Education \& Improvement Wales, Wales, UK

Email: drmbashir@gmail.com

Keywords: Aorta, Aortic arch, Dissection, Acute dissection, Frozen elephant trunk, Meta-analysis

Funding declarations: None to be declared

Conflicts of interest: None to be declared

I have read with great interest, "Neurological complications following frozen elephant trunk for aortic dissection: What's truly to blame?"(1) . Dr. Tan's comments on our article, "Hypothermic circulatory arrest 
time affects neurologic outcomes of frozen elephant trunk for acute type A aortic dissection: A systematic review and meta-analysis" published in the Journal of Cardiac Surgery (2).

Frozen Elephant Trunk (FET) was described primarily to treat arch and proximal descending aorta pathologies in a single-stage approach in early 2000s (3) and subsequently, evolved with great endeavours through recent years. The most important advantage of FET is to provide "hybrid" alternative treatment for complex pathologies which required two morbid surgeries, formerly. The FET technique has been used in different aortic pathologies, acute aortic dissection (Stanford Type A and B, DeBakey I), atherosclerotic arch, descending or thoraco-abdominal aneurysms and for other pathologies such as penetrating aortic ulcers (4).

Although outstanding improvements were achieved after introduction of FET, one of the main serious drawback of this approach is potential association with neurologic events. The observed discrepancy among rate of neurologic outcomes including transient or permanent spinal cord injury (SCI) and stroke after FET deployment in recent reports is principally attributed to individual cerebral perfusion methods and cerebral protection techniques. The incidence rate of permanent or transient ischemic SCI after conventional ET has been reported between $0.4 \%$ and $2.8 \%$ in previous reports (5). In contrast, the incidence of SCI after FET ranged between $8 \%$ in multicenter studies (6) and $20 \%$ in single-center reports (7). In addition, several contributors (i.e., length of the device and distal circulatory arrest time) have been proposed to explain SCI and stroke observed after FET procedure, mainly derived from observational studies on patients with diverse aortic pathologies.

Individual pathologies are inherent with specific risk of post-operative outcomes after FET procedure, so we decided to adjudicate the pooled estimated rate of neurologic outcomes in a specific subgroup of patients diagnosed with acute type A aortic dissection (ATAAD). The present meta-analysis, including 35 studies with 3211 patients diagnosed with ATAAD, revealed that the FET procedure in this subset of patients was associated with $3 \%(95 \%$ CI $2-4)$ and 5\% (95\% CI 4-7) of postoperative SCI and stroke, respectively. These results are comparable with recent studies.

Preventza et al explored the role of device length on SCI rate in the published meta-analysis included all patients who underwent total arch replacement with FET and concluded that with the extension of stent beyond T8 is associated with higher risk of SCI (8). In spite of the fact that surgical methods and stent variations would affect the outcomes after procedure, intra-operative measures such as HCA time are of paramount. In a review article, Gupta et al concluded that there are still debates on the definition of safe HCA time (9). Some endeavors have been applied to define the type of HCA duration as mild, moderate, or deep HCA by experts in the field of aortic surgery (9). Future randomized and prospective studies are required to address this issue and provide us an appropriate length of HCA for aortic surgeries, and also show us how we can attenuate neurologic events by some modifications in surgical modalities, particularly HCA time.

We hypothesized that HCA time is one of major indicator of adverse neurologic outcomes in FET course of action. The univariate meta-regression of included patients showed significant relationships between the HCA time and the development of postoperative stroke $(\mathrm{p}=0.04)$ and SCI $(\mathrm{p}=0.05)$. These findings could be a backbone of future studies involved in devices' design and modifications in FET deployment during surgery. As we revealed in recent meta-analysis that the anastomosis in Zone 2 was associated with a lower rate of renal failure compared with Zone 3 (10), this approach might improve neurologic outcomes by reducing HCA time.

However, the present meta-analysis had several limitations. The studies analyzed were observational in design, with the inherent bias and the lack of uniformity in surgical methodology and reporting. The absence of a standard reporting schema for total arch replacement using FET, some important data are missing, which incapacitates us to perform subgroup analysis. Future individual patient's meta-analysis and/or prospective randomized reports can help us with the identification of contributors to the neurologic outcomes and the appraisal of HCA time.

\section{References}


1. Tan SZCP, El Santawy H, Abdelhaliem A. Neurological complications following frozen elephant trunk for aortic dissection: What's truly to blame? Journal of Cardiac Surgery.n/a(n/a).

2. Mousavizadeh M, Daliri M, Aljadayel HA, Idhrees M, Rezaei Y, Bashir M, et al. Hypothermic Circulatory Arrest Time affects neurological outcomes of Frozen Elephant Trunk for Acute Type A Aortic Dissection: a systematic review and meta-analysis. 2021.

3. Karck M, Chavan A, Hagl C, Friedrich H, Galanski M, Haverich A. The frozen elephant trunk technique: a new treatment for thoracic aortic aneurysms. The Journal of Thoracic and Cardiovascular Surgery. 2003;125(6):1550-3.

4. Hickey GL, Dunning J, Seifert B, Sodeck G, Carr MJ, Burger HU, et al. Statistical and data reporting guidelines for the European Journal of Cardio-Thoracic Surgery and the Interactive CardioVascular and Thoracic Surgery. Oxford University Press; 2015.

5. Etz CD, Plestis KA, Kari FA, Luehr M, Bodian CA, Spielvogel D, et al. Staged repair of thoracic and thoracoabdominal aortic aneurysms using the elephant trunk technique: a consecutive series of 215 first stage and 120 complete repairs. European journal of cardio-thoracic surgery. 2008;34(3):605-15.

6. Jakob H, Tsagakis K, Pacini D, Di Bartolomeo R, Mestres C, Mohr F. The International E-vita Open Registry: data sets of 274 patients. J Cardiovasc Surg (Torino). 2011;52(5):717-23.

7. Flores J, Kunihara T, Shiiya N, Yoshimoto K, Matsuzaki K, Yasuda K. Extensive deployment of the stented elephant trunk is associated with an increased risk of spinal cord injury. The Journal of thoracic and cardiovascular surgery. 2006;131(2):336-42.

8. Preventza O, Liao JL, Olive JK, Simpson K, Critsinelis AC, Price MD, et al. Neurologic complications after the frozen elephant trunk procedure: A meta-analysis of more than 3000 patients. The Journal of thoracic and cardiovascular surgery. 2020;160(1):20-33. e4.

9. Gupta P, Harky A, Jahangeer S, Adams B, Bashir M. Varying evidence on deep hypothermic circulatory arrest in thoracic aortic aneurysm surgery. Texas Heart Institute Journal. 2018;45(2):70-5.

10. Rezaei Y, Bashir M, Mousavizadeh M, Daliri M, Aljadayel HA, Mohammed I, et al. Frozen elephant trunk in total arch replacement: A systematic review and meta-analysis of outcomes and aortic proximalization. Journal of Cardiac Surgery. 2021. 